\title{
Erratum to: Rheumatoid arthritis: what has changed?
}

\author{
Edith Villeneuve $\cdot$ Paul Emery
}

Published online: 13 October 2010

(C) ISS 2010

Erratum to: Skeletal Radiol

DOI 10.1007/s00256-008-0579-4

Unfortunately, the affiliations listed for Paul Emery were not correct. They should read:

1. Section of Musculoskeletal Disease, Leeds Institute of Molecular Medicine, University of Leeds, Leeds, UK

2. NIHR Leeds Musculoskeletal Biomedical Research Unit, Leeds Teaching Hospitals Trust, Leeds, UK

The online version of the original article can be found at http://dx.doi. org/10.1007/s00256-008-0579-4.

E. Villeneuve $(\bowtie) \cdot$ P. Emery

Section of Musculoskeletal Disease,

Leeds Institute of Molecular Medicine, University of Leeds,

Leeds, UK

e-mail: villeed@hotmail.com

P. Emery

NIHR Leeds Musculoskeletal Biomedical Research Unit,

Leeds Teaching Hospitals Trust,

Leeds, UK 